\title{
Original Research Article \\ Correlation between optic disc changes and visual field changes in glaucoma suspects: A clinical study
}

\author{
Authors
}

\section{Dr Nivedita Choudhury ${ }^{1}$, Dr Dinesh Kumar Bhagat ${ }^{2 *}$, Dr Anirban Chakrabarti ${ }^{3}$}

${ }^{1}$ Assistant Professor, Department of Ophthalmology, Mata Gujri Memorial Medical College \& Lions Seva Kendra

Hospital, Kishanganj, Bihar- 855107, India

${ }^{2}$ Assistant Professor, Department of Ophthalmology, Mata Gujri Memorial Medical College \& Lions Seva Kendra

Hospital, Kishanganj, Bihar- 855107, India

${ }^{3}$ Associate Professor, Department of Psychiatry, Mata Gujri Memorial Medical College \& Lions Seva Kendra

Hospital, Kishanganj, Bihar- 855107, India

*Corresponding Author

\section{Dr Dinesh Kumar Bhagat}

Assistant Professor, Department of Ophthalmology, Mata Gujri Memorial Medical College \& Lions Seva Kendra

Hospital, Kishanganj, Bihar- 855107, India

\begin{abstract}
Background: This study was done to identify a group of POAG suspects from the patients attending the O.P.D and to monitor them periodically for a period of one year for detecting early signs of glaucomatous damage of ONH and glaucomatous VFDs.
\end{abstract}

Materials \& Methods: The present study was carried out in Mala Gujri Memorial Medical College, Department of Ophthalmology, Kishanganj from July $15^{\text {th }}, 2016$ to June $14^{\text {th }}$, 2017. About 50 glaucoma suspects were selected for the study from the patients attending the O.P.D. The selection was made on the basis of findings of gonioscopy, applanation tonometry, direct ophthalmoscopy, slit lamp biomicroscopy, stereoscopic visualization of ONH by $+90 D$ lens, automated perimetry by Humphrey Field analyzer (central 30-2 test) and fundus photography.

Results: Of the 100 eyes of 50 cases, suspicious ONH changes were found in 45 eyes of 24 glaucoma suspects, suspicious VFDs in 25 eyes of 18 glaucoma suspects and IOP $>21 \mathrm{~mm}$ of $\mathrm{Hg} /$ suspicious diurnal variation test \&/or suspicious provocative test in 30 eyes of 20 glaucoma suspects. Of the 45 eyes of 24 glaucoma suspects with ONH changes, bilateral ONH changes were seen in 21 cases; rest 3 cases had unilateral ONH changes. Pallor of NRR and diffuse thinning of NRR was found in 44.4\%, splinter hemorrhage in $11.1 \%$, notching of NRR in $11.1 \%$ and vertically oval cup in 33.3\%. Regarding correlation of asymmetry of C/D with VFDs in glaucoma suspects, of the 50 glaucoma suspects, asymmetry of C/D was found in 19 persons, of which asymmetry of $0.1-0.3$ in 12 persons and $>0.3$ were found in 7 persons. Of the 19 glaucoma suspects with asymmetric C/D, VFDs were seen in 20 eyes of 14 persons (6 persons had bilateral VFDs, 8 persons had unilateral VFDs). VFD percentage of $83.3 \%$ was noted in C/D asymmetry between 0.1-0.3 and 142.9\% when asymmetry was >0.3. So increasing asymmetry of $C / D$ was related to increasing percentage of VFDs.

Conclusion: The nature of VFDs correlated with ONH changes in established POAG. Localized field defects were seen in early and moderate ONH changes and more generalized field defects were revealed in advanced ONH changes. The nature of VFDs revealed the typical arrangement of nerve fibers in the retina and the optic nerve and the typical pattern of glaucomatous nerve damage. Regarding correlation of C/D with IOP in established POAG, an increase of $C / D$ with increase in IOP was observed.

Keywords: Primary open angle glaucoma, ocular hypertension, glaucoma suspect, optic disc changes, visual field changes. 


\section{Introduction}

Primary open angle glaucoma suspect and related entities are included in ICD \#10 H40. The American Academy of Ophthalmology; Preferred Practice Patterns Glaucoma Panel used the term glaucoma suspect to mean primary open angle glaucoma suspect. ${ }^{2}$ Glaucoma of all types constitutes the second most common cause of legal blindness. POAG is the major health problem. It is important to identify the risk factors and monitor the glaucoma suspects earlier and in an accurate way so that the glaucoma related blindness can be prevented by intervention at the earliest sign of glaucomatous damage. ${ }^{3}$

The manifestations of glaucoma range from mechanical angle closure of outflow structures in patients with angle closure glaucoma (ACG), who typically present with ocular pain and acute visual loss, to increased resistance of outflow in patients with open angle glaucoma (OAG), who are often asymptomatic. Although glaucoma embodies a diverse group of diseases, all these diseases share common characteristics, the hallmarks of which include progressive irreversible damage to the optic nerve head and the retinal ganglion cells with corresponding visual field loss. ${ }^{3,4}$ Ocular hypertension (OHT) is defined as IOP $>22 \mathrm{mmHg}$ (2 standard deviations above the mean), but without any other abnormal features in the optic discs, VFs, or RNFL. ${ }^{5}$

\section{Aims and Objectives}

1) To identify a group of POAG suspects from the patients attending the O.P.D and to monitor them periodically for a period of one year for detecting early signs of glaucomatous damage of $\mathrm{ONH}$ and glaucomatous VFDs.

2) To find out the percentage of different ONH changes in POAG suspects.

3) To find out the percentage of various VFDs in the POAG suspects.

4) To find out the relation among various $\mathrm{ONH}$ changes, IOP and VFDs in established POAG detected from the POAG suspects.

\section{Materials \& Methods}

The present study was carried out in Mala Gujri Memorial Medical College, Department of Ophthalmology, Kishanganj from July $15^{\text {th }}, 2016$ to June $14^{\text {th }}, 2017$. About 50 glaucoma suspects were selected for the study from the patients attending the O.P.D. The selection was made on the basis of findings of gonioscopy, applanation tonometry, direct ophthalmoscopy, slit lamp biomicroscopy, stereoscopic visualization of $\mathrm{ONH}$ by $+90 \mathrm{D}$ lens, automated perimetry by Humphrey Field analyzer (central 30-2 test) and fundus photography.

\section{Selection Criteria}

The presences of one or more of the following criteria were used for selection of glaucoma suspects $^{6}$ :

1. a) Intraocular pressure (IOP) more than 21 $\mathrm{mm}$ of $\mathrm{Hg}$ on more than one occasion. (b) diurnal fluctuation of IOP $>5 \mathrm{~mm}$ of $\mathrm{Hg}$ and I or provocative test showing fluctuations of $\mathrm{IOP}>5 \mathrm{~mm}$ of $\mathrm{Hg}$. c) asymmetry of the IOP between the two eyes of $5 \mathrm{~mm}$ of $\mathrm{Hg}$ or more by diurnal variation test and /or provocative test.

2. Suspicious optic disc changes ${ }^{7}$ : (a) Cup: disc ratio $>0.5$, especially in vertical axis $b$ ) Asymmetry of disc cup $>0.2 \mathrm{C} / \mathrm{D}$ c) Disc hemorrhage (superficial splinter hemorrhage) (d) Diffuse or focal notching of the disc rim, especially at the inferior and superior poles e) Vertically oval cup (f) Pallor neuroretinal rim (NRR) (g) Thinning of NRR.

(a) Suspicious visual field ${ }^{7}$ : (a) generalized depression. (b) baring of blind spots d) Nasal step $<10^{\circ}$ (d) relative scotoma $<5^{\circ}$

(b) Presence of one or more of the associated risk factors in persons having $\mathrm{ONH}$ changes and /or VFDs and/or IOP>21 mm of $\mathrm{Hg}$ such as strong family history of glaucoma, age above 40 years, myopia, systemic hypertension, diabetes mellitus, cardiovascular diseases, migraine, and peripheral vasospasm.

In this study, the term glaucoma suspect is used to 
mean primary open angle glaucoma suspect. Gonioscopy was done to select the cases with open angle.

\section{Exclusion Criteria}

1) Angle closure glaucoma

2) Secondary open angle glaucoma

3) Non-cooperative patients

4) Patients on any drug or operative procedure that can affect IOP

5) Those who were attending hospital irregularly.

The study group was followed up every 3 monthly intervals for a period of one year and on each follow up, gonioscopy, applanation tonometry, direct ophthalmoscopy, slit lamp biomicroscopy, stereoscopic examination of $\mathrm{ONH}$ by $+90 \mathrm{D}$ lens and automated perimetry were done. Any progression of $\mathrm{ONH}$ changes, and/or VFDs and /or alteration of IOP were noted. Fundus photography was done if definite progression of ONH changes were found. Data were analyzed using standard statistical procedures.

\section{Results}

Of the 100 eyes of 50 cases, suspicious $\mathrm{ONH}$ changes were found in 45 eyes of 24 glaucoma suspects, suspicious VFDs in 25 eyes of 18 glaucoma suspects and IOP $>21 \mathrm{~mm}$ of $\mathrm{Hg} /$ suspicious diurnal variation test \&/or suspicious provocative test in 30 eyes of 20 glaucoma suspects. Of the 45 eyes of 24 glaucoma suspects with $\mathrm{ONH}$ changes, bilateral $\mathrm{ONH}$ changes were seen in 21 cases; rest 3 cases had unilateral $\mathrm{ONH}$ changes. Pallor of NRR and diffuse thinning of NRR was found in $44.4 \%$, splinter hemorrhage in $11.1 \%$, notching of NRR in $11.1 \%$ and vertically oval cup in $33.3 \%$.

Regarding correlation of asymmetry of C/D with VFDs in glaucoma suspects, of the 50 glaucoma suspects, asymmetry of C/D was found in 19 persons, of which asymmetry of $0.1-0.3$ in 12 persons and $>0.3$ were found in 7 persons. Of the 19 glaucoma suspects with asymmetric C/D, VFDs were seen in 20 eyes of 14 persons (6 persons had bilateral VFDs, 8 persons had unilateral VFDs). VFD percentage of $83.3 \%$ was noted in C/D asymmetry between $0.1-0.3$ and $142.9 \%$ when asymmetry was $>0.3$. So increasing asymmetry of $\mathrm{C} / \mathrm{D}$ was related to increasing percentage of VFDs.

Regarding relation of C/D with VFDs in glaucoma suspects, eyes with C/D 0.2 had no VFDs, there was gradual increase in VFDs with increasing C/D, VFDs were $100 \%$ when C/D 0.7 and above. Of the 100 eyes of glaucoma suspects, 25 eyes of 18 persons had VFDs. Seven persons had bilateral VFDs, and 11 persons had unilateral VFDs. Of the 25 eyes with VFDs, enlargement of blind spot was seen in $8 \%$,paracentral, Seidel, arcuate and double arcuate scotomas in $40 \%$, Roenne's central nasal step in $8 \%$, localized or generalized field constriction in $40 \%$. Defect could not be studied (atypical VFDs) in 4\%. Of the 100 eyes of 50 glaucoma suspects, 28 eyes of 18 persons had IOP $>21 \mathrm{~mm}$ of $\mathrm{Hg}$ on more than one successive occasions. Of the 18 persons with raised IOP, 10 persons had IOP> $21 \mathrm{~mm}$ of $\mathrm{Hg}$ on both eyes and the rest 8 persons had raised IOP in one of the eyes.

Of the rest 72 eyes (of 32 persons of bilateral IOP $<21 \mathrm{~mm}$ of $\mathrm{Hg}$ and 8 persons of unilateral IOP $<21 \mathrm{~mm}$ of $\mathrm{Hg}$ ), positive provocative test (Water Drinking Test) showing fluctuation > 5 $\mathrm{mm}$ of $\mathrm{Hg}$ and positive diurnal variation showing IOP fluctuation $>5 \mathrm{~mm}$ of $\mathrm{Hg}$ in one eye each of 2 different persons were found. About 9 eyes of 7 persons ( 2 persons with bilateral and 5 persons with unilateral involvement) with raised IOP/positive provocative test and /or diurnal variation and $\mathrm{ONH}$ changes, 3 eyes of 2 persons (1 person with bilateral involvement and 1 person with unilateral involvement) with raised IOP/ positive provocative test and /or diurnal variation associated with VFDs and 8 eyes of 4 persons (bilateral involvement) with raised IOP/positive provocative test and diurnal variation only (no associated $\mathrm{ONH}$ changes and/or VFDs) were found. About 10 eyes of 8 persons ( 2 persons with bilateral involvement and 6 persons with unilateral involvement) with raised IOP $>21 \mathrm{~mm}$ of $\mathrm{Hg} /$ positive provocative test and/or diurnal variation with $\mathrm{ONH}$ changes and VFDs were 
found. These glaucoma suspects were established as POAG.

Regarding percentage of eyes with raised IOP/positive provocative test and/or diurnal variation and correlation with $\mathrm{ONH}$ and VFDs, raised IOP with ONH changes was found in $30 \%$, raised IOP with VFDs in 10\%, raised IOP only in $26.7 \%$ and raised IOP, ONH changes and VFDs in $33.3 \%$ eyes. Regarding risk factors, of the 50 glaucoma suspects, risk factors were present in 43 persons. Multiple risk factors were also present in the same person in $45 \%$. Age > 40 years was present in $23 \%$, family history of POAG in $25 \%$, myopia> 1D in $20 \%$, diabetes mellitus in $15 \%$ and systemic hypertension in 5\%. No glaucoma suspect was found with migraine, cardiovascular diseases and peripheral vasospasm hence no association could be established.

Of the $45 \%$ glaucoma suspects with multiple risk factors, age $>40$ years and family history of POAG was $23 \%$, age $>40$ years and family history of POAG and myopia>-1D was $10 \%$, diabetes mellitus with family history of POAG was $2 \%$ and age $>40$ years with diabetes mellitus with family history of POAG was $10 \%$. Of the 50 selected glaucoma suspects, POAG was established in only 8 persons during the follow-up period. All the 8 established cases of POAG had multiple risk factors.

Of the 8 established cases of POAG (total 10 eyes), laminar dot sign with saucerization was found in $20 \%$, bayoneting of disc edge with saucerization in $30 \%$, pallor of NRR, thinning of NRR, notching of NRR \& peripapillary chorioretinal atrophy with laminar dot sign with baring of circumlinear vessels with bayoneting at disc edge in $40 \%$ and nasal shifting of vessels with laminar dot sign with saucerization in $1 \%$.

In each of the established case of POAG, typical glaucomatous VFDs were present; so, the association between ONH changes in POAG and VFDs was $100 \%$. Regarding correlation of C/D with IOP in established POAG, the following observations were made:eyes with IOP ranging 21-30 $\mathrm{mm}$ of $\mathrm{Hg}$ had mean C/D 0.55, eyes with IOP ranging 31-40 $\mathrm{mm}$ of $\mathrm{Hg}$ had mean C/D 0.75.
When IOP was above $41 \mathrm{~mm}$ of $\mathrm{Hg}$. mean C/D was 0.9 . So, there was an increase of $C / D$ with increase in IOP.

\section{Discussion}

\section{Margin in Glaucoma Suspects}

According to Drance et $\mathrm{al}^{8}$, splinter hemorrhages, usually near the margin of the optic nerve head, mostly in the inferior quadrant, are a common feature of early glaucomatous damage. In the present study splinter hemorrhage at the disc margin was found in $11.1 \%$ cases.

CUP-Disc Asymmetry Ano Correlation with VFD in Glaucoma Suspects

Annaly MF (1967) ${ }^{9}$ showed that the physiological cup tends to be symmetrical between the two eyes of the same individual and a C/D of more than 0.2 between the two eyes of the same individual occurred only in $1 \%$ of the populations. In the present study, out of the 50 glaucoma suspects, asymmetry of C/D was found in 19 persons, of which asymmetry of $0.1-0.3$ in 12 persons and $>0.3$ were found in 7 persons. Of the 19 glaucoma suspects with asymmetric C/D, VFDs were seen in 20 eyes of 14 persons ( 6 persons had bilateral VFDs, 8 persons had unilateral VFDs). VFD percentage of $83.3 \%$ was noted in C/D asymmetry between $0.1-0.3$ and $142.9 \%$ when asymmetry was $>0.3$.

\section{C/D \& VFDs in Glaucoma Suspects \& Establshed POAG}

According to Shields ${ }^{1}$, when the discs in normal population was studied by direct ophthalmoscopy, the distribution was found to be non-Gaussian with most eyes a C/D of $0.0-0.3$ and only $1-2 \%$ being 0.7 or greater, when stereoscopic views were utilized, a Gaussian distribution was found with a mean C/D of 0.4 and approximately 5\% had C/D of 0.7. Yablonski ME et $\mathrm{al}^{10}$ (1960) showed that an increase in C/D is an important predictor of glaucomatous VFDs. Gloster pointed out the relationship between the vertical $\mathrm{C} / \mathrm{D}$ and the percentage of VFDs. ${ }^{11}$ There was an increase in the prevalence of VFOs increased with C/D and was significantly increased when the vertical C/D was over 0.7. Armaly ${ }^{9}$ (1967) and Drance et al ${ }^{12}$ 
(1978) reported that increased C/D is an important predictor of glaucomatous VFDs. The present study with 100 eyes of 50 glaucoma suspects found that eyes with C/D 0.2 had no VFDs. There was gradual increase in VFDs with increasing C/D. VFDs were $100 \%$ when C/D 0.7 and above. The present study detected 10 eyes of 8 established case of POAG which had mean vertical $\mathrm{C} / \mathrm{D}$ ranging from $0.55-0.9$. VFDs were present in $100 \%$. Thus, the present study corroborates with the results of previous studies.

\section{Visual Field Changes in Glaucoma Suspects}

In the present study with 100 eyes of 50 glaucoma suspects, 25 eyes of 18 persons had VFDs. Seven persons had bilateral VFOs, 11 persons had unilateral VFDs. Enlargement of blind spot was seen in $8 \%$, paracentral, Seidel, arcuate and double arcuate scoromas in $40 \%$, Roenne's central nasal step in $8 \%$, localized or generalized field defect in $40 \%$. Defect could not be studied (atypical defects) in 4\%. In the present study, we have used Humphrey Automated Field Analyzer to detect the changes in the field of vision of the glaucoma suspects. Central 30-2 Threshold Test was utilized with SITA-standard strategy. Quigley HA, Sommer A (1995) in a comparative study of VFDs in glaucoma using manual Goldmann Perimetry and Automated Static Perimetry concluded that Automated Static Perimetry detects VFDs earlier than manual Goldmann Perimetry. ${ }^{13}$

\section{Correlation between ONHs and VFOs in established POAG:}

The present study detected 8 established cases of POAG where typical glaucomatous ONH changes were present with typical VFDs. So the association of $\mathrm{ONH}$ changes with VFDs in established POAG was found to be $100 \%$. Study of relevant literature reveals that in most patients with glaucoma, clinically recognizable $\mathrm{ONH}$ changes precede VFDs and the presence or absence of glaucomatous VFDs can usually but not always, be predicted from the appearance of the $\mathrm{ONH}^{5}$

Quigley et al (1982) ${ }^{14}$ attempted to correlate axon loss in the ONH with the VFDs. Although limited by small sample size their works did suggest that. not only does nerve fiber loss occur prior to reproducible field defect in some patients with elevated IOP, but the extent of axonal loss may be much more than the corresponding field change. In addition to the fact that presence of $\mathrm{ONH}$ cupping predicts the presence of VFDs, the nature of ONH cupping can be used to predict the type of VFDs. Extensive or focal loss of neural tissue, especially in the superior or inferior poles is the most reliable indicator of VFDs and is usually associated with a field defect in the corresponding arcuate area. In some cases, field loss occurred before the pallor reaches the disc margin. ${ }^{5}$

Garway-Heath DF, Poinoosawmy D, Fitzke FW, Hitchings RA (2000) ${ }^{15}$ showed that a clinically useful map that relates visual field test points to regions of the $\mathrm{ONH}$ has been produced. The map will aid clinical evaluation of glaucoma patients and suspects, as well as form the basis for investigations of the relationship between retinal light sensitivity and ONH structure. The studies of Schuman J, Hee MR, Puliafito D et $\mathrm{al}^{16}$ using advanced techniques like Optical coherence Tomography (OCT) showed high degree of correlation of nerve fiber layer thickness with VFDs detected by Humphrey Automated Field Analyzer.

The present study with 100 eyes of 50 glaucoma suspects revealed 8 established cases of POAG in which $\mathrm{ONH}$ changes can be correlated with the VFDs and thus establishes the fact that VFDs in glaucoma correlate with the damage to the neurons in retina with reflection of their characteristic arrangement within the retina and within the optic nerve. These findings were documented by showing the VFDs by automated static perimetry and corresponding $\mathrm{ONH}$ changes were shown by fundus photograph of the same case. Thus, results of the present study corroborate with previous studies.

\section{Correction between IOP and Optic Disc Changes in Glaucoma Suspects}

In the present study, 28 eyes of 18 persons with IOP $>21 \mathrm{~mm}$ of $\mathrm{Hg}$ and one eye each of positive provocative test and diurnal variation of 2 different persons were found. Nine eyes (of 7 persons) with 
raised IOP (including positive provocative test and/or diurnal variation) associated with $\mathrm{ONH}$ changes, 3 eyes (of 2 persons) with raised IOP (including positive provocative test and/or diurnal variation) associated with VFDs and 8 eyes (of 4 persons) with raised IOP/ positive provocative test and/or diurnal variation only were found. Ten eyes of 8 persons ( 2 persons with bilateral and 6 persons with unilateral manifestations) had raised IOP/ positive provocative test and/or diurnal variation, ONH changes and VFDs. These were established POAG. Tanito $\mathrm{M}$, et $\mathrm{al}^{17}$ in their study "Correlation between intraocular pressure level and optic disc changes in high-tension glaucoma suspects" concluded that the IOP level correlates with topographic changes in the optic disc in eyes of patients suspected of having high-tension glaucoma.

\section{Correlation between IOP and Optic Disc Changes in Established POAG}

In the present study, 10 eyes of 8 established cases of POAG showed increase in vertical mean C/D with increase in IOP. Pohjanpelto PE et al (1974) revealed in his study with 307 patients, the prevalence of $\mathrm{ONH}$ damage each pressure group. ${ }^{18} \mathrm{He}$ concluded that with increasing IOP, there was increased incidence of nerve fiber damage there by leading to VFDs. Thus, the present study corroborates with the results of previous studies.

\section{Importance of Risk Factors in Glaucoma Suspects}

Glaucoma suspect may have one or more ocular and /or systemic risk factors that may lead to the development of POAG. ${ }^{6}$ The strong risk factors include increasing age ${ }^{18}$, strong family history ${ }^{6}$, African American descent ${ }^{19}$, elevated IOP, thinner central corneal thickness (in Ocular Hypertension Treatment study, the cut off mark was taken to be $556 \mu \mathrm{m}$ but it varies in different population ${ }^{20}$ ), larger than average pattern standard deviation index on otherwise normal, baseline threshold visual field examinations (> $1.98 \mathrm{~dB}, 30-2$ test, Humphrey perimeter). ${ }^{20}$ Systemic hypertension, Cardiovascular disease, myopia, migraine and peripheral vasospasm have been implicated as possible risk factors in the development of glaucomatous optic nerve damage. ${ }^{21,22,23}$

In the present study, of the 50 glaucoma suspects, risk factors were present in 43 persons. Multiple risk factors were also present in the same person in $45 \%$. Age > 40 years was present in $23 \%$, family history of POAG in $25 \%$, myopia> $1 \mathrm{D}$ in $20 \%$, diabetes mellitus in $15 \%$ and systemic hypertension in $5 \%$. No glaucoma suspect was found with migraine, cardiovascular diseases and peripheral vasospasm hence no association could be established. Gordon MO, et $\mathrm{al}^{20}$ in their study concluded that increasing age is a definite risk factor for POAG. Bankes JL (1968) in his study of 3000 individuals from general population found that the prevalence of POAG increases with age. ${ }^{24}$ Several studies revealed strong family history of POAG is a definite risk factor. POAG is said to have a genetic basis and the inheritance is likely to be polygenic and multifactorial. The Baltimore Eye Survey ${ }^{25}$ found that the relative risk of having glaucoma is increased 3.7-fold for individuals who have siblings with POAG. Family history of glaucoma in a sibling is the greatest risk factor, followed by glaucoma in a parent. Myopia is another risk foctor. ${ }^{22}$ The prevalence of POAG is more among the myopics as well as an increased frequency of myopia is seen among those who have POAG, ocular hypertension and normal tension glaucoma. ${ }^{5}$ A systemic review and metaanalysis published online in 2014 concluded increased risk of POAG in individuals with diabetes mellitus. ${ }^{26}$

\section{Conclusion}

The following conclusion can be drawn from the present study:

The commonest presenting feature of glaucoma suspect is ONH changes, followed by raised IOP and VFDs. There was an increase in the percentage of VFDs with increase in asymmetry in the $C / D$ between the two eyes. There was a gradual increase in the percentage of VFDs with increasing $\mathrm{C} / \mathrm{D}$, and when the ratio was 0.7 or more, all the eyes had VFDs. The nature of VFDs revealed localized as well as generalized defects. 
The maximum percentage of VFDs were localized defects like paracentral scotoma, Siedel, arcuate and double arcuate scotomas, equalized by localized or generalized field constriction $(40 \%$ each) and followed by nonspecific changes like enlargement of blind spot. There is a significant role of risk factors in glaucoma suspects. In those glaucoma suspects who had multiple risk factors, POAG was confirmed. Thus, we can come to the conclusion that the presence of one or more of the risk factors in glaucoma suspects increases the vulnerability of glaucomatous optic nerve damage.

\section{References}

1. H40-H42 - ICD-10: H40.0, Glaucoma suspect. Ocular hypertension. H40.1, Primary open-angle glaucoma. Available at:

https://apps.who.int/classifications/apps/ic d/icd10online2003/gh40.htm.

2. Prum BE Jr, Lim MC, Mansberger SL, Stein JD, Moroi SE, Gedde SJ, et al. Primary Open-Angle Glaucoma Suspect Preferred Practice Pattern(®) Guidelines. Ophthalmology. 2016 Jan;123(1):P112-51.

3. Pan Y, Varma R. Natural history of glaucoma. Indian J Ophthalmol. 2011;59 Suppl (Supp11):S19-S23.

4. Foster PJ. The epidemiology of primary angle closure and associated glaucomatous optic neuropathy. Semin Ophthalmol. 2002; 17:50-8.

5. Hodapp E, Parrish RK, Anderson DR. 1st ed. US: St Louis Mosby; 1993. Clinical Decisions in Glaucoma.

6. Ahmad SS. Glaucoma suspects: A practical approach. Taiwan J Ophthalmol. 2018;8(2):74-81.

7. Spaeth GL, Henderer J, Liu C, et al. The disc damage likelihood scale: Reproducibility of a new method of estimating the amount of optic nerve damage caused by glaucoma. Trans Am Ophthalmol Soc 2002; 100:181-185.

8. Drance SM, Flairclough M, Butler, DM, Kottler MS. The importance of disc hemorrhage in the prognosis of chronic open angle glaucoma. Arch Ophthalmol. 1977 Feb;95(2):226-8.

9. Armaly MF. Genetic determination of cup/disc ratio of the optic nerve. Arch Ophthalmol. 1967 Jul;78(1):35-43.

10. Yablonski ME, Zimmerman TJ, Kass MA, Becker B. Prognostic significance of optic disk cupping in ocular hypertensive patients. Am J Ophthalmol. 1980 Apr; 89(4):585-92.

11. Gloster J. Quantitative relationship between cupping of the optic disc and visual field loss in chronic simple glaucoma. $\mathrm{Br} \quad \mathrm{J}$ Ophthalmol. 1978 Oct;62(10):665-9.

12. Susanna R, Drance SM. Use of discriminant analysis I. Prediction of visual field defects from features of the glaucoma disc. Arch Ophthalmol. 1978 Sep;96(9):1568-70.

13. Katz J, Tielsch JM, Quigley HA, Sommer A. Automated perimetry detects visual field loss before manual Goldmann perimetry. Ophthalmology. 1995 Jan;102(1):21-6.

14. Quigley HA. Glaucoma's optic nerve damage: changing clinical perspectives. Ann Ophthalmol. 1982 Jul;14(7):611-2.

15. Garway-Heath DF, Poinoosawmy D, Fitzke FW, Hitchings RA. Mapping the visual field to the optic disc in normal tension glaucoma eyes. Ophthalmology. 2000 Oct;107(10):1809-15.

16. Schuman JS, Pedut-Kloizman T, Hertzmark E, Hee MR, Wilkins JR, Coker JG, Puliafito CA, Fujimoto JG, Swanson EA. Reproducibility of nerve fiber layer thickness measurements using optical coherence tomography. Ophthalmology. 1996 Nov;103(11):1889-1898.

17. Tanito M, Itai N, Dong J, Ohira A, Chihara E. Correlation between intraocular pressure level and optic disc changes in high-tension glaucoma suspects. Ophthalmology. 2003 May;110(5):915-21. 
18. Pohjanpelto PE, Palva J. Ocular hypertension and glaucomatous optic nerve damage. Acta Ophthalmol (Copenh). 1974;52(2):194-200.

19. Tielsch JM, Sommer A, Katz J, Royall RM, Quigley HA, Javitt J. Racial variations in the prevalence of primary open-angle glaucoma. The Baltimore Eye Survey. JAMA. 1991 Jul 17;266(3):36974.

20. Gordon MO1, Beiser JA, Brandt JD, Heuer DK, Higginbotham EJ, Johnson $\mathrm{CA}$, et al. The Ocular Hypertension Treatment Study: baseline factors that predict the onset of primary open-angle glaucoma. Arch Ophthalmol. 2002 Jun;120(6):714-20; discussion 829-30.

21. Leske MC, Connell AM, Wu SY, Hyman LG, Schachat AP. Risk factors for openangle glaucoma. The Barbados Eye Study. Arch Ophthalmol. 1995 Jul;113(7):918-24.

22. Mitchell P, Hourihan F, Sandbach J, Wang JJ. The relationship between glaucoma and myopia: the Blue Mountains Eye Study. Ophthalmology. 1999 Oct;106(10):2010-5.

23. The Advanced Glaucoma Intervention Study (AGIS): 3. Baseline characteristics of black and white patients. Ophthalmology. 1998 Jul;105(7):1137-45.

24. Bankes JL, Perkins ES, Tsolakis S, Wright JE. Bedford glaucoma survey. Br Med J. 1968;1:791-796.

25. Sommer A, Tielsch JM, Katz J, et al. Relationship between intraocular pressure and primary open angle glaucoma among white and black Americans. The Baltimore Eye Survey. Arch Ophthalmol. 1991 Aug. 109(8):1090-5.
26. Zhou M, Wang W, Huang W, Zhang X. Diabetes mellitus as a risk factor for openangle glaucoma: a systematic review and meta-analysis. PLoS One. 2014 Aug 19;9(8):e102972. 